Post-print version (peer reviewed, author accepted manuscript), please cite the published article as follows:

Fernandes, T. P., Shaqiri, A., Brand, A., Nogueira, R. L., Herzog, M. H., Roinishvili, M., Santos, N. A. \& Chkonia, E. (2019). Schizophrenia patients using atypical medication perform better in visual tasks than patients using typical medication. Psychiatry research, 275, 31-38.

DOI: https://doi.org/10.1016/i.psychres.2019.03.008

The article is under copyright by Oxford University Press, the publisher should be contacted for permission to re-use or reprint the material in any form.

\title{
Schizophrenia patients using atypical medication perform better in visual tasks than patients using typical medication
}

Thiago P. Fernandes ${ }^{\mathrm{a}}$; Albulena Shaqiri ${ }^{*}$; Andreas Brand ${ }^{\mathrm{c}}$; Renata L. Nogueira ${ }^{\mathrm{d}}$; Michael H. Herzog $^{\text {b; }}$ Maya Roinishvilie,f; Natanael A. Santos ${ }^{\text {a }}$; Eka Chkonia ${ }^{\mathrm{e}}$

aPerception, Neuroscience and Behaviour Laboratory, Federal University of Paraiba, Joao Pessoa, Brazil

${ }^{b}$ Laboratory of Psychophysics, Brain Mind Institute, Ecole Polytechnique Fédérale de Lausanne (EPFL), Lausanne, Switzerland

'Institute for Psychology and Cognition Research, University of Bremen, Bremen, Germany

${ }^{\mathrm{d}}$ Federal University of Pernambuco, Recife, Brazil

eDepartment of Psychiatry, Tbilisi State Medical University, Tbilisi, Georgia

${ }^{\mathrm{f}}$ Department of Behaviour and Cognitive Functions, I. Beritashvili Institute of Physiology, Tbilisi, Georgia

* Corresponding author: Albulena Shaqiri, Laboratory of Psychophysics, Brain Mind Institute, School of Life Sciences, Ecole Polytechnique Fédérale de Lausanne, Station 19, CH-1015, Lausanne, Switzerland; tel: (+41) 21-693-1741, fax: (+41) 21-69-31749, e-mail: albulena.shaqiri@epfl.ch 


\section{Introduction}

Schizophrenia (SCZ) is a debilitating disorder affecting about $1.0 \%$ of the population worldwide (Jablensky, 2010; Mathers and Loncar, 2006). Deficits of SCZ patients are ample, including many cognitive and perceptual functions. One of the most important questions is to what extent medication influences or even causes these deficits. This question is not only important for schizophrenia research but also for neuroscience in general because it addresses the question how drugs modify brain processing. Here, we investigated how medication influences performance in visual and, to a secondary extent, in cognitive tasks.

A major challenge of antipsychotic medication is to reduce positive symptoms, caused by dopaminergic hyperfunction in mesolimbic pathways, and negative symptoms, caused by dopaminergic hypofunction in mesocortical pathways, without drastically reducing neurotransmission (Brisch et al., 2014). Antipsychotic drugs are usually subdivided into typical (or first-generation) and atypical (or second-generation) drugs. Typical medication causes extrapyramidal side effects and tardive dyskinesia, which is not the case for atypical drugs. Each antipsychotic drug has its own receptor specificity. Characteristic for typical antipsychotics is their strong affinity to dopaminergic $D_{2}$ receptors, whereas atypical antipsychotics have a lower affinity to $\mathrm{D}_{2}$ receptors acting on a spectrum of other receptors, such as e.g. 5-HT (Meltzer, 2017; Li et al., 2016).

In general, SCZ patients perform worse than healthy controls in most visual tests, including contrast detection (Cadenhead et al., 2013; Fernandes et al., 2018d; Kiss et al., 2010; Shoshina et al., 2015, Slaghuis, 1998;) and visual backward masking (Braff, 1981; Chkonia et al., 2010; Green et al. 1994; review: Herzog and Brand, 2015). Very little is known to what extent the visual deficits depend on medication. In particular, there are only a few studies that investigated the effects of typical vs. atypical medication on contrast detection (for a review, see Silverstein, 2016). Kéri et al., (2002) found that the higher the dosage of typical antipsychotic medication, the stronger impaired was contrast detection $(n=20)$. Chen et al., (2003) found that contrast detection was worse in typical $(n=8)$ than atypical $(n=25)$ medicated patients with the latter performing at about the same level as healthy controls $(n=39)$. Unmedicated patients $(n=6)$ performed even better than healthy controls. Cadenhead et al., (2013) also found that unmedicated patients ( $n=5)$ performed better than controls $(n=53)$. Shoshina et al., (2014) found that contrast detection was worse in typical medicated patients $(n=20)$ for low spatial frequencies, but not for medium spatial 
frequencies, for which atypically medicated patients $(n=25)$ performed worse. It needs to be noted that the sample sizes were very small in these studies.

In visual backward masking, SCZ patients perform in general worse than healthy controls (Braff, 1981; Green et al., 1994; Chkonia et al., 2010). Comparing unmedicated and medicated SCZ patients, Brody et al., (1980; $n=6$ unmedicated, $n=6$ medicated patients, respectively), Braff et al., (1982; $n=16 ; n=20)$, Butler et al., (1996; $n=7 ; n=7)$, and Cadenhead et al., $(1997 ; n=14 ; n=76)$ did not find any significant differences of medication on backward masking. Again, sample sizes are small.

Here, we investigated whether typical medication has different effects than atypical medication on contrast detection, vernier duration and backward masking. Patients were very carefully selected. In Study 1, conducted in Brazil, we assessed contrast sensitivity and three cognitive measures (Stroop Test, Trail Making Test B and Flanker Test). Out of a large pool of 158 patients, we selected 50 patients, who had clear-cut either typical or atypical single drug medication (patients were using the same class of antipsychotics for at least five years). In Study 2, conducted in Georgia, we assessed vernier duration, backward masking and two cognitive measures (Wisconsin Card Sorting Test and Continuous Performance Test). From a pool of 276 SCZ patients, we included 97 patients, who took either typical or atypical medication. Some patients, however, took more than one antipsychotic drug.

In both studies, patients also performed cognitive tests. SCZ patients usually suffer from a broad range of cognitive deficits (meta-analysis: Fioravanti et al., 2012; but see Buchsbaum et al., 1990, for the Continuous Performance Test). Some studies explored the effects of antipsychotic medication on cognition in schizophrenia. Results are mixed ranging from no effect (see Harvey et al. 1990; Liu et al., 2000 for CPT) to improvements for medicated patients (Nestor et al., 1991; Orzack et al., 1967). Several studies compared the medication effects of typical and atypical drugs with mixed results again. Some studies reported that atypical medication resulted in better neurocognitive performance than typical medication (Désaméricq et al., 2014; Hagger et al., 1993; Harvey and Keefe, 2001; Keefe et al., 2007a; Lee et al., 1997; Woodward et al., 2005). In contrast, Keefe et al., (2007b) showed in the CATIE study that atypical medication had no superior effects to perphenzine. Since data of patients treated with one drug is scarce, we report performance in the cognitive tests here as secondary results because the data may be of interest, for example, for meta-analysis. We do not propose that there is a direct link between perception and cognition.

To preface our results, we found a reduction in visual performance for all SCZ patients compared to controls in line with almost all studies. Patients taking atypical 
antipsychotics performed better in all three visual tasks than patients with typical antipsychotics. We found similar trends for the cognitive tasks, where patients taking atypical performed slightly (but not significantly) better than patients taking typical antipsychotics.

\section{Study 1: Contrast sensitivity and cognitive tests}

\subsection{Material and methods}

\subsubsection{Participants}

Fifty healthy controls (HCs; mean age $=35.4$ years, $S D=8.05$ years), 25 patients who were diagnosed with SCZ and using typical antipsychotics (mean age $=35.1$ years, $S D=8.14$ years), and 25 patients who were diagnosed with SCZ and using atypical antipsychotics (mean age $=38.4$ years, $S D=7.46$ years) were recruited from the Psychosocial Care Center. Psychiatrists at the same institution diagnosed SCZ according to the Diagnostic and Statistical Manual of Mental Disorders (DSM-5; American Psychiatric Association, 2013). The exclusion criteria for SCZ patients were: (a) presence of neurological disorders, such as intellectual disability, (b) presence of comorbidities, such as depression or multiple diagnoses, (c) changes in medication in the last 6 months, (d) discontinuation of medication at any time, and (e) chronic heavy smoking (Fernandes et al., 2018c) as assessed by the Fagerström Test for Nicotine Dependence (score > 6; Heatherton et al., 1991). This information was obtained from a continuous study of the medical records (Fernandes et al., 2018d).

The following typical antipsychotics were used: haloperidol $(n=19)$, chlorpromazine $(n=4)$, and levomepromazine $(n=2)$. The following atypical antipsychotics were used: quetiapine $(n=7)$, olanzapine $(n=8)$, risperidone $(n=7)$, clozapine $(n=2)$, and ziprasidone $(n=1)$. The SCZ patients in each group (typical and atypical) used the same class of antipsychotic for at least five years (some of them used the same class since the firstepisode).

The HC were staff members at the Federal University of Paraiba and recruited through newspaper advertisements. The HCs had no neuropsychiatric disorders according to the Structured Clinical Interview for the DSM (SCID; American Psychiatric Association, 2015). The global exclusion criteria were: $<25$ old or $>48$ years old, current history of neurological disorder, cardiovascular disease, history of head trauma, history of contact with substances such as solvents, current or previous drug or substance abuse, and current use of medications that may affect visual processing and cognition (e.g. benzodiazepines). 
Participants had no retinal abnormalities on fundoscopic examination or optical coherence tomography. All of the observers were had normal or corrected-to-normal (20/20) vision as determined by a Snellen chart. All participants were matched for gender, age, and level of education.

\subsubsection{Procedure}

First, we conducted the neuropsychological tests. This procedure lasted 1 hour 30 minutes and was performed in a quiet and comfortable room. In a second testing, participants were tested in CSF from 30 to 45 minutes. In addition, and to avoid fatigue, participants were encouraged to take breaks at their discretion.

\subsubsection{Contrast sensitivity}

Stimuli and apparatus. Stimuli were presented on a 19-inch LG CRT monitor with $1024 \times 786$ resolution and a refresh rate of $100 \mathrm{~Hz}$. Stimuli were generated using a VSG 2/5 video card (Cambridge Research Systems Ltd., Rochester, UK), which was run on a Precision T3500 computer with a W3530 graphics card. The average luminance was 50 $\mathrm{cd} / \mathrm{m}^{2}$. All of the procedures were performed in a room at $26^{\circ} \mathrm{C} \pm 1^{\circ} \mathrm{C}$, with the walls covered in gray to better control luminance during the experiments. Measurements were performed with binocular vision at a distance of $150 \mathrm{~cm}$ from the computer monitor. Monitor luminance and chromatic calibrations were performed using a ColCAL MKII photometer (Cambridge Research Systems Ltd., Rochester, UK). Contrast sensitivity was determined by the Metropsis software (Cambridge Research Systems Ltd., Rochester, UK). The stimuli were linear, vertically oriented sine-wave gratings with spatial frequencies of 0.2, 0.5, 1.0, 2.0, 5.0, 10.0, and 20.0 cycles per degree (cpd). The gratings were equiluminant of 5 degrees of visual angle (see Figure 1) and were presented on the monitor at $2.5^{\circ}$ from the central fixation cross (for stimulus details, see Fernandes et al., 2017; Fernandes et al., 2018a, 2018c).

Prior to the start of the tests, detailed task instructions were provided. Accuracy was emphasized over speed. A practice session was performed to familiarize participants with the procedure and to avoid misunderstandings about the task. The Metropsis software incorporates a check on the validity of the data by using catch trials to detect random responding. The participants easily understood the task. Participants were instructed to maintain fixation on a small black fixation cross in the center of the monitor. A twoalternative forced-choice (2-AFC) method was used. The participants' task was to identify, 
using a remote control response box, whether the grating was presented on either the left or right side of the computer screen (Figure 1). The order of the spatial frequencies was randomized within a session.

A three-down one-up logarithmic staircase with dynamic steps was used to derive a contrast threshold with a level of accuracy of target detection of $79.4 \%$ on a psychometric function (Levitt, 1971). Initially, the contrast values appeared at the suprathreshold level, for which we expected correct responses. Thus, after three consecutive correct responses, contrast decreased by $.7 \mathrm{~dB}$. After every incorrect response, contrast increased by $1.0 \mathrm{~dB}$. Each stimulus had an exposure time of $600 \mathrm{~ms}$. After responding, the next trial started after $300 \mathrm{~ms}$.

The session ended after 12 contrast reversals occurred. Higher values indicate better performance (for procedural details, see Fernandes et al., 2017a; Fernandes et al., 2017b Fernandes et al., 2018c).

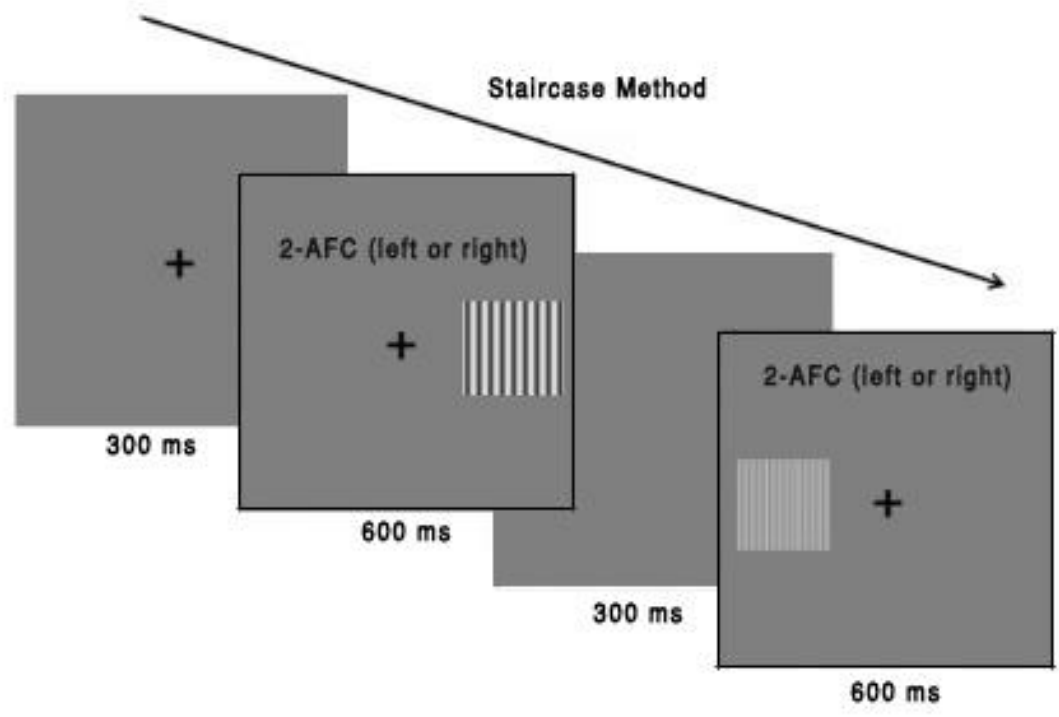

Figure 1. Contrast sensitivity function task. The task was to identify, using a remote control response box, whether the gratings were presented either on the left or right side of the computer screen. Each stimulus had an exposure time of $600 \mathrm{~ms}$, with an inter-trial interval of $300 \mathrm{~ms}$. The Metropsis algorithm randomizes spatial frequencies (low, medium and high) and contrast values. 


\subsubsection{Cognitive measures}

Stroop Color-Word Interference (Stroop, 1935). This test was used to measure executive function, such as attention, cognitive flexibility, inhibition, and information processing speed. A series of color words was presented. Participants were asked to name the color of the words instead of reading the word. Conflict occurs when the color of the word and the name are different. We used four colors (red, blue, yellow, and green) in several combinations that were randomly displayed on a computer screen one at time, sequentially. The dependent measure was the reaction time (in seconds) for the incongruent minus congruent stimuli (Bugg, 2008).

Flanker Task (Eriksen and Eriksen, 1974). This task was used to evaluate attentional control and inhibition. Stimuli (letters, such as ZXYQ) were centrally presented and flanked by peripheral stimuli. We used reaction time as a measure of cognitive ability. A faster reaction time indicated better performance.

Trail Making Test B (TMT-B) (Tombaugh, 2004). This test was used to assess cognitive operations, such as visual search, psychomotor speed, cognitive flexibility, and sustained attention. The participant was presented with a sheet of randomly placed circles and instructed to draw a line that connected numbers and letters in correct ascending sequence. Fewer errors and a faster reaction time indicated better performance. We computed the reaction time (in seconds) for the completion time as the dependent variable.

\subsubsection{Statistical analyses}

Distributions for each group were compared using the Monte Carlo methods for skewness and kurtosis (Antonius, 2003; Tabachnick, Fidel, 2007). The cutoff value was > 1.96 for both kurtosis and skewness. Outliers were transformed using the Fractional Rank method (applying inverse cumulative distribution functions; Beasley, Erickson, and Allison, 2009). We analyzed the data of the CSF by a multivariate analysis with the groups as between subjects factor and the spatial frequencies as dependent variables. For further analysis, we calculated posthoc comparisons with Bonferroni corrections. The assumption of variance homogeneity was not violated. For the cognitive measures, separate ANOVAs with post hoc comparisons were performed. For categorical variables (gender), the chi-squared test was used. Independent $t$-tests were used to compare clinical data between both SCZ groups (e.g., duration of illness, CPZ, BPRS). Pearson's product-moment correlation $(r)$ and the point-biserial correlation $(r)$ were used to assess relationships between CSF data and biosociodemographic variables (e.g., age, gender, level of education). Effect sizes $>.50$ were considered medium-to-large. 


\subsection{Results}

\subsubsection{Sample characteristics}

Characteristics of participants are summarized in Table 1. The groups did not differ in age, level of education, and the ratio of males to females. A $3 \times 2$ chi-square was performed to compare the differences between males and females, and the results indicated no significant differences, $\chi=5.78, p=0.056$. In addition, no differences on the illness duration, BPRS and $\mathrm{CPZ}$ were found among SCZ patients.

Table 1: Sample characteristics

\begin{tabular}{|c|c|c|c|c|}
\hline Variable & $\begin{array}{c}\text { HC } \\
(n=50)\end{array}$ & $\begin{array}{l}\text { SCZ typical } \\
(n=25)\end{array}$ & $\begin{array}{c}\text { SCZ atypical } \\
(n=25)\end{array}$ & $P$ \\
\hline \multicolumn{5}{|l|}{ Gender } \\
\hline Male & 26 & 18 & 18 & $.445^{a}$ \\
\hline Female & 24 & 7 & 5 & $.001^{a}$ \\
\hline \multicolumn{5}{|l|}{ Age } \\
\hline Age, years $(S D)$ & $36.1(7.3)$ & $35.8(7.5)$ & $36.3(7.0)$ & $.689^{b}$ \\
\hline Level of Education, years $(S D)$ & $11.4(2.1)$ & $9.0(2.5)$ & $8.7(2.1)$ & $.433^{\mathrm{b}}$ \\
\hline Age of onset, years (SD) & - & $19.8(2.0)$ & $20.6(3.3)$ & $.303^{\#, \mathrm{~b}}$ \\
\hline Duration of illness, years $(S D)$ & - & $15.5(2.6)$ & $13.9(3.1)$ & $.556^{4, \mathrm{~b}}$ \\
\hline Number of hospitalizations & - & $6.6(4.2)$ & $6.4(2.7)$ & $.891^{\#, \mathrm{~b}}$ \\
\hline Comorbidities & - & - & - & - \\
\hline Brief Psychiatric Rating Scale score & - & $42.5(7.4)$ & $45.0(8.2)$ & $.187^{4, \mathrm{~b}}$ \\
\hline Hamilton Rating Scale for Depression & $1.8(1.2)$ & $7.59(1.5)$ & $6.50(1.5)$ & $.318^{i, \mathrm{~b}}$ \\
\hline Stroop (incongruent), seconds & $1.06(.04)$ & $3.64(.70)$ & $3.11(.14)$ & $.001^{n, \mathrm{~b}}$ \\
\hline Flanker task, seconds & $.71(.31)$ & $1.03(.20)$ & $1.14(.27)$ & $.085^{\mathrm{a}}$ \\
\hline Trail-Making Test-B & $45.11(10.4)$ & $57.81(11.7)$ & $51.32(14.1)$ & $.080^{\sharp, \mathrm{b}}$ \\
\hline \multicolumn{5}{|l|}{ Illness Duration } \\
\hline $0-5$ years & - & 6 & 8 & \\
\hline $6-10$ years & - & 6 & 7 & \\
\hline$>10$ years & - & 13 & 10 & \\
\hline CPZ & - & $615(412)$ & $478(396)$ & $.882^{\#, \mathrm{~b}}$ \\
\hline
\end{tabular}

*Statistically significant difference. "Between schizophrenia groups. ${ }^{a} \chi 2$ test. ${ }^{b}$ Mann-Whitney U-test. 


\subsubsection{Contrast sensitivity function}

There were significant differences in the CSF between groups $\left(F_{14,182}=9.230, p<\right.$ 0.001; Pillai's trace $=0.985$, partial $\omega 2=0.427$ [95\% CI: 0.286 to 0.552 ]) for all spatial frequencies (Figure 2).

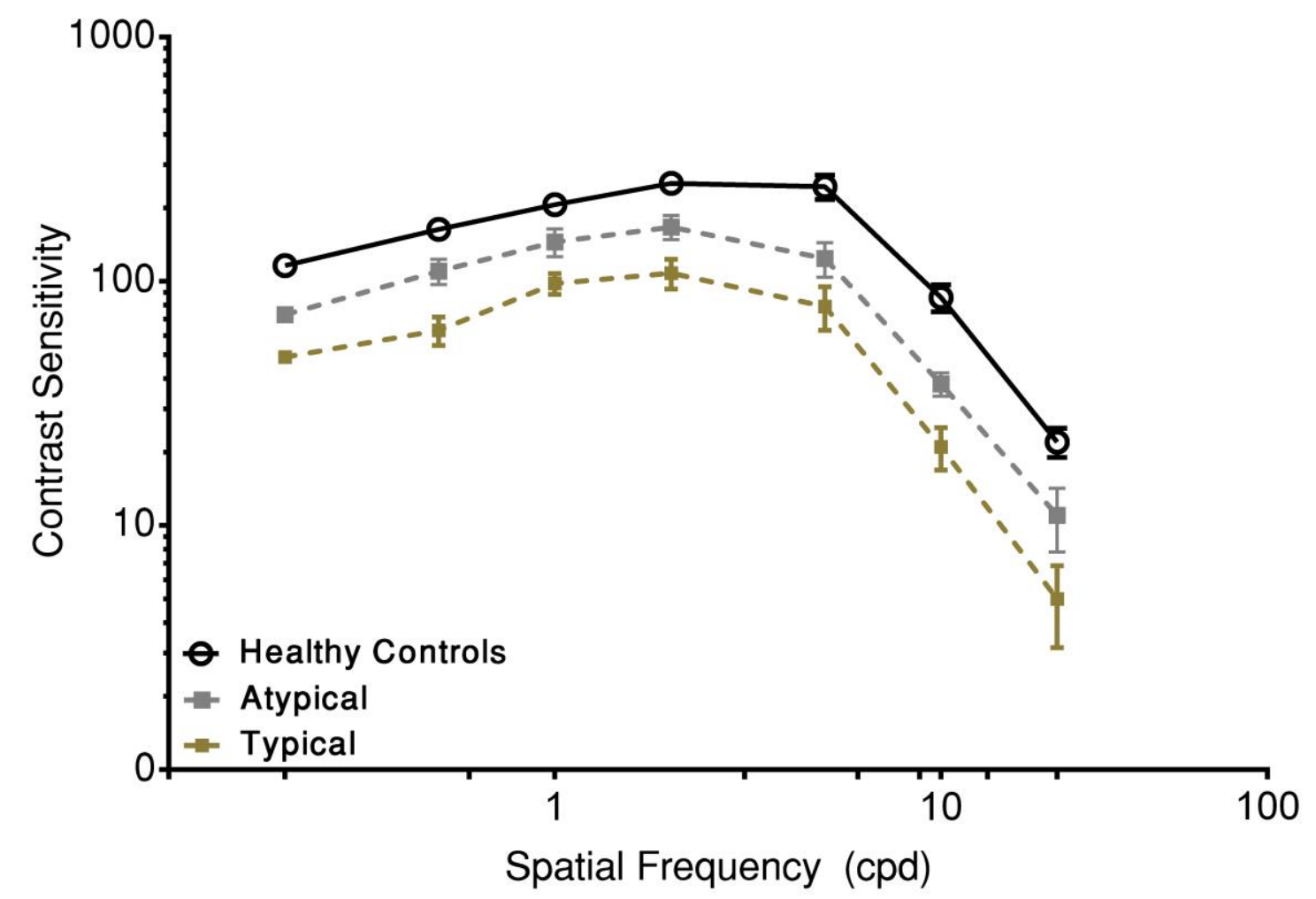

Figure 2. Contrast sensitivity as a function of spatial frequency (cpd) in healthy controls and schizophrenia patients taking atypical and typical antipsychotics. The data are expressed as the mean sensitivity (reciprocal of contrast threshold). Error bars represent the deviations of the mean sensitivity

Post-hoc test revealed statistically significant differences for the CSF of all spatial frequencies $(p<0.001)$. Both SCZ groups had lower contrast sensitivity than HCs (patients taking typical antipsychotics: all $p$-values $<0.001$; patients taking atypical antipsychotics: all $p$-values < 0.001). SCZ patients taking typical antipsychotics had lower contrast sensitivity than those with atypical drugs (all $p$-values $<0.01$ ).

\subsubsection{Cognitive tests}

The results for the cognitive measures are summarized in Figure 3. There were no statistically significant differences between the groups in the Flanker task $\left(F_{2,97}=0.516, p=\right.$ 
$0.559)$, the Stroop Test $\left(F_{2,97}=0.748, p=0.343\right)$ and in the Trail-Making Test-B $\left(F_{2,97}=\right.$ $2.037, p=0.136)$.
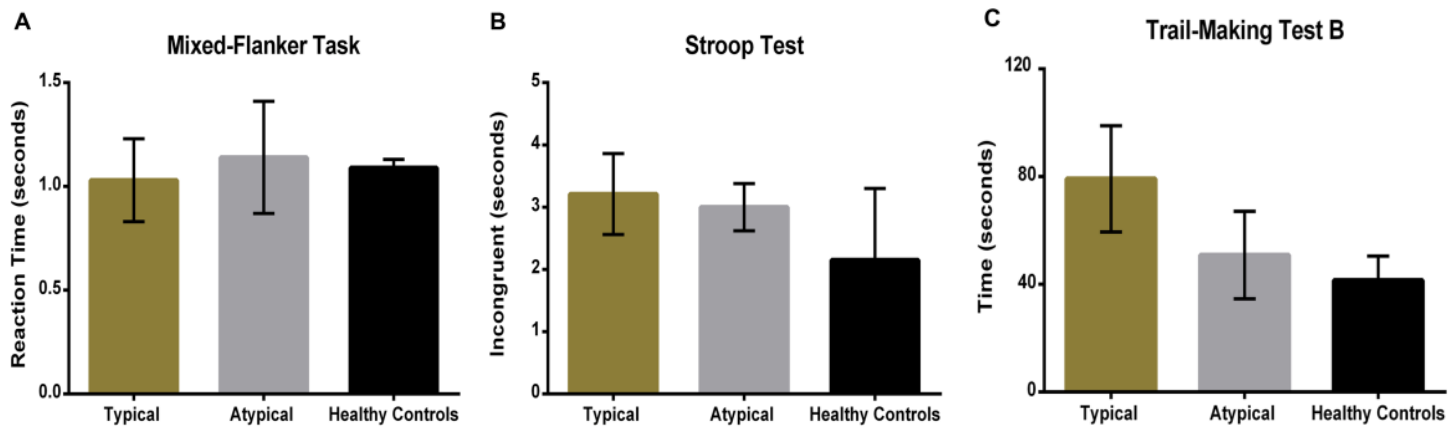

Figure 3. Differences on neurocognitive performance between the groups. (A) Mean reaction time (s) for the Mixed-Flanker task; (B) Mean reaction time (s) for the Stroop task comparing differences in incongruent responses; $(\mathbf{C})$ The average Trail-Making Test-B completion time.

\subsubsection{Correlation analysis}

Separate correlation analyses were conducted to investigate relationships between the demographic data and the CSF results. No significant relationships between any of the pairs of variables were found in both the control and the patient group (all $p$-values > 0.05). There were no significant correlations between CPZ equivalents and CSF.

\section{Study 2: Visual backward masking and cognitive tests}

\subsection{Materials and methods}

\subsubsection{Participants}

Hundred and fifty-eight HCs (mean age $=34.7$ years, $S D=8.8$ years), $49 \mathrm{SCZ}$ patients taking typical antipsychotics (mean age $=38.2$ years, $S D=7.9$ years) and $48 \mathrm{SCZ}$ patients taking atypical antipsychotics (mean age $=35.4$ years, $S D=8.8$ years) were recruited from the Asiatani psychiatric hospital or the rehabilitation centre or outpatients of the Gotsiridze psychoneurological dispensary. Patients were diagnosed according to DSM-4 by means of an interview based on the SCID, information of the staff, and the study of the records. Psychopathology of SCZ patients was assessed by an experienced psychiatrist (EC) by Scales for the Assessment of Negative Symptoms and Scales for the Assessment of Positive Symptoms (SANS: Andreasen, 1984; SAPS: Andreasen, 1984). Healthy controls were recruited from the general population. Patients were part of a large database that contains participants who took part in different studies, some of which are already published (Chkonia 
et al., 2010; Roinishvili et al., 2015; Shaqiri et al., 2015). We have never investigated how medication could affect performance on the different visual and cognitive tests.

General exclusion criteria were drug or alcohol abuse (Fernandes et al., 2018c), neurological or other somatic illnesses that might possibly impair cognitive functioning. All participants had normal or corrected-to-normal vision, with a visual acuity of $\geq 0.8$ (corresponding to 20/25) at least in one eye, as determined with the Freiburg Visual Acuity Test (Bach, 1996). Participants were no older than 55 years.

We included patients with either typical antipsychotic medication or atypical medication. Fifty-four patients received one single antipsychotic drug, 37 patients used two antipsychotics, and two patients received three antipsychotics. Each included patient took either typical or atypical antipsychotic medication. Patients with a mixture of typical and atypical drugs were excluded. Typical antipsychotic drugs were haloperidol, trifluoperazine, chlorpromazine, fluphenazine, clopenthixol, sulpirid, triphtazine, levomepromazine. Atypical drugs were clozapine, olanzapine, risperidone, quetiapine, amisulprid.

Most patients received additional drugs $(n=68)$, in most cases trihexyphenidyl against Parkinson symptoms $(n=46)$, others took mood stabilizers or antidepressants. Patients taking benzodiazepines were excluded.

\subsubsection{Procedure}

\subsubsection{Visual processing}

The shine-through paradigm is described in detail in Herzog et al., (2004) and Chkonia et al., (2010). In the first step, verniers, two vertical white bars, were presented on a black background. The lower bar was slightly offset either to the right or left (the direction was chosen randomly). Observers were asked to indicate this offset direction by button press. In this step, we determined the shortest vernier duration (VD) in order to perform Vernier offset discrimination below 40". The starting VD was of $150 \mathrm{~ms}$ and we reduced durations until the Vernier offset was above 40" (4A). In the second step, a grating followed the Vernier. The grating comprised 25 or 5 Verniers without offset of the same length as the target Vernier. Conditions were presented in blocks of 80 trials. After the Vernier, an interstimulus-interval (ISI) followed, i.e., a blank screen, and then the grating for $300 \mathrm{~ms}$ (Figure 4B). We adaptively assessed the target-mask stimulus-onset-asynchrony (SOA) which yields a performance level of $75 \%$ correct responses. The results are plotted as SOA = VD + ISI. For more details see Herzog et al., (2004) and Chkonia et al., (2010). 
A

B

VD

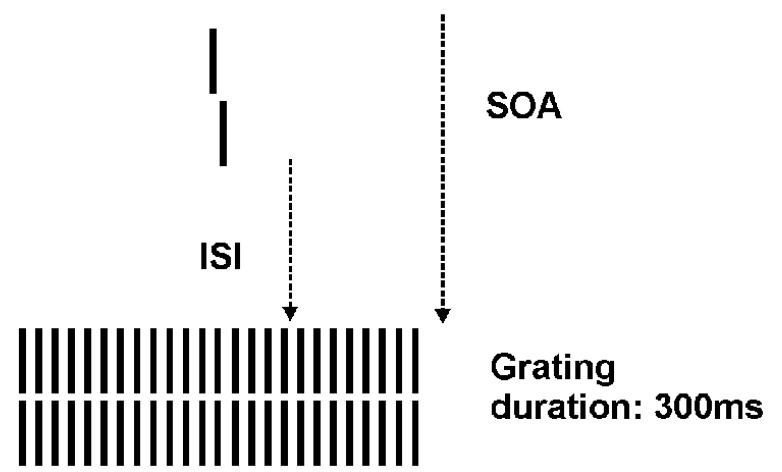

Figure 4. (A) We first presented a Vernier alone (A) and as a second step (B), this Vernier was followed by a blank screen (ISI) and a grating comprised of 25 (or 5, not presented) aligned Verniers (SOA $=\mathrm{VD}+\mathrm{ISI})$.

\subsubsection{Cognitive tests}

We administered a computerized version of the Nelson Test (Nelson, 1976), a modified Wisconsin Card Sorting Test (WCST) with 48 cards. This test is assumed to assess executive functions. We also tested participants with the degraded Continuous Performance Test (CPT; 240 digits, 10\% targets, degradation 40\%) with a duration of 4 minutes. Observers had to detect the pair " $1-9$ ", which means that they had to press a button when the digit 9 was presented just after the digit 1 . The digits were presented randomly with a rate of one per second with a presentation time of $50 \mathrm{~ms}$. For a detailed description of the used tests see Chkonia et al. (2010).

\subsubsection{Statistical analyses}

For gender as a categorical variable, the chi-squared test was used. The other data (demographical and psychopathological, backward masking, CPT and WCST) was analyzed by separate ANOVAs with post hoc tests using a Bonferroni correction. The effect size of the differences in backward masking, CPT and WCST was calculated as Cohen's $d$ and values > .50 were considered as medium-to-large effects. Correlations were calculated as bivariate Pearson's product-moment correlation $(r)$.

\subsection{Results}

\subsubsection{Sample characteristics}


The sample characteristics are summarized in Table 2. All three groups differed in age mainly driven by the difference between HCs and the atypical medication group. The three groups were also different with regard to the level of education, the ratio of males and females and visual acuity. However, between the groups with typical and atypical drugs, respectively, there were no significant differences with regard to age, years of education, illness duration, SANS, SAPS, visual acuity or CPZ.

A $3 \times 2$ chi-square was performed to compare the differences between males and females, and the results indicated significant differences, $\chi=8.50, p=0.015$.

Table 3: Sample characteristics for Study 2.

\begin{tabular}{lllll}
\hline & $\begin{array}{l}\text { HC } \\
(\mathbf{n = 1 5 8})\end{array}$ & $\begin{array}{l}\text { SCZ } \\
\text { (typical } \\
\text { antipsychotics) } \\
(\mathbf{n = 4 9 )}\end{array}$ & $\begin{array}{l}\text { SCZ } \\
\text { (atypical } \\
\text { antipsychotics) } \\
(\mathbf{n = 4 8})\end{array}$ & \\
& & & & \\
\hline Gender & 27 & 37 & $0.001^{* \mathrm{a}}$ \\
Male & 85 & 22 & 11 & $0.001^{* \mathrm{a}}$ \\
Female & 73 & & & \\
\hline
\end{tabular}

Age

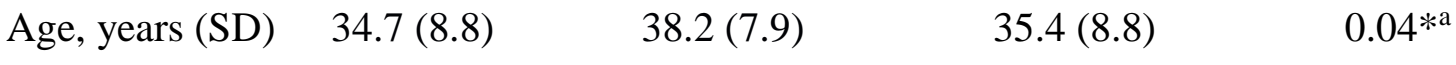

$\begin{array}{lllll}\text { Level of } & 15.0(2.9) & 12.8(2.8) & 13.6(2.5) & 0.001 * \mathrm{a}\end{array}$

education,

years (SD)

Illness duration

$12.5(9.1)$

$12.6(8.1)$

$0.78^{\#, \mathrm{~b}}$

$\begin{array}{lllll}\text { Visual acuity } & 1.6(0.4) & 1.4(0.3) & 1.5(0.4) & 0.10^{\mathrm{a}}\end{array}$

SANS

$10.7(6.0)$

$10.5(5.6)$

$0.59^{\#, \mathrm{~b}}$

SAPS

$10.1(3.1)$

$10.5(10.8)$

$0.16^{\#, \mathrm{~b}}$ 


\subsubsection{Visual tests}

For the visual tests, we found significant differences for all variables (VD: $F[2,252]=42.74$, $p<0.001$; SOA25: $F[2,252]=61.4, p<0.001$; SOA5: $F[2,252]=54.75, p<0.001$; see Figure 5). These significances are mainly due to the difference between controls and patients. Post hoc comparisons between the typical and the atypical group of SCZ patients, however, were significantly different only for VD ( $p<0.0001$, Cohen's $d=0.68)$, SOA25 $(p=0.03$, Cohen's $d=0.34$ ) and SOA5 ( $p=0.003$, Cohen's $d=0.37$ ). There were no significant post hoc effects for the CPT and the WCST between patients with typical vs. atypical drugs.

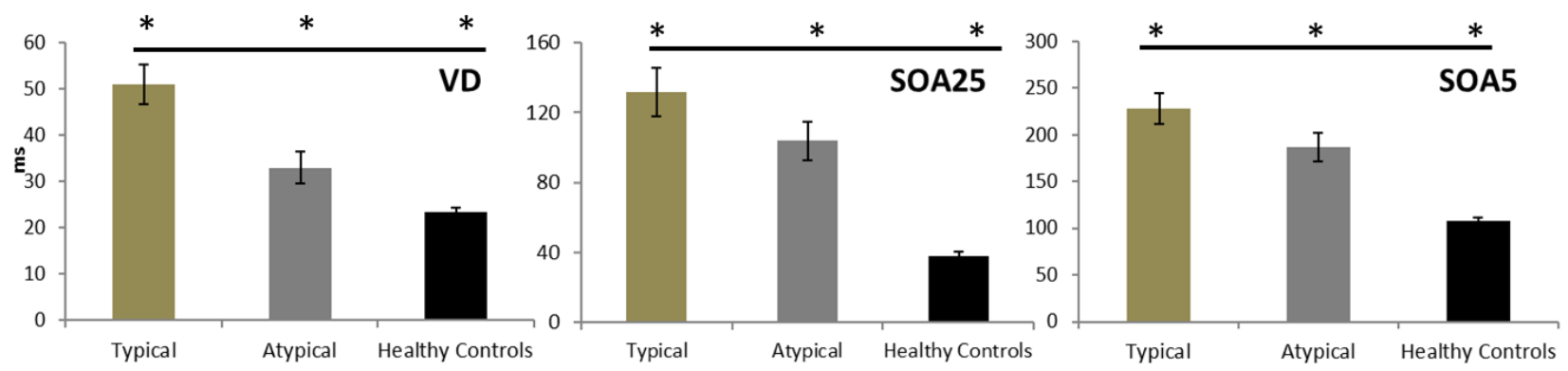

Figure 5. Performance on VD, SOA 25 and SOA5. All three tests revealed significant differences between typical and atypical patients as well as controls.

\subsubsection{Cognitive tests}

In the WCST and the CPT, there were statistically significant differences between the controls and the patients (CPT: $F[2,243]=18.91, p \leq 0.0001$; WCST: $F[2,251]=10.52, p \leq$ 0.0001), such that controls performed better in the CPT and had fewer errors in the WCST test (Figure 6). Post hoc analysis revealed that for the WCST, both groups of SCZ patients performed at the same level $(p=0.6)$ and the typical or atypical medication did not have an effect on the performance on the CPT either $(p=0.55)$. 

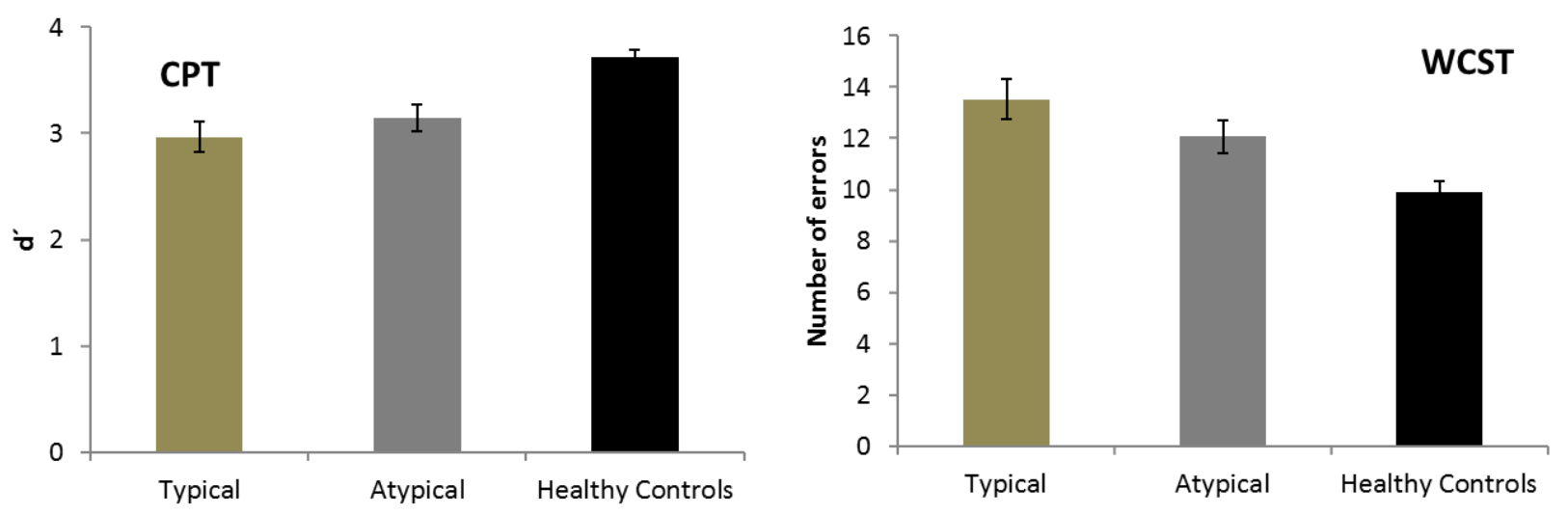

Figure 6. Performance on cognitive tests (CPT and WCST) is higher in patients treated with atypical than with typical drugs. These differences, however, are not significant. Patients performed differently than healthy controls.

\subsubsection{Correlation analysis}

WCST errors showed a significant negative correlation with education, not all other tests correlated with education, neither in patients nor in controls. There were no significant correlations of performance with CPZ. Some of the patients with typical antipsychotics received trihexyphenidyl against Parkinson symptoms $(n=34)$. Comparing both subgroups, there were no effects on performance in the visual tasks, however.

\section{Discussion}

We investigated how typical and atypical antipsychotics influence vision and, to a secondary extent, cognition. For contrast detection, patients with atypical antipsychotics had superior performance than patients treated with typical medication for all spatial frequencies. Similarly, we found that patients with atypical medication performed better in vernier duration and visual backward masking than patients with typical medication. We did not observe any influence of symptom severity (using BPRS, SANS, SAPS) and CPZ equivalents on visual performance. For the cognitive tests, we found only trends but they were always in the same direction: atypical treatment led to better results than typical medication.

We can only speculate why patients with atypical medication perform better than those with typical antipsychotics because there are no reliable data for drug free patients. Cadenhead et al., (2013) and Chen et al., (2003) found that unmedicated patients performed better than healthy controls in contrast detection. However, the sample sizes for the unmedicated patients ( $n=5, n=6$, respectively) were extremely small. In backward masking, 
SCZ patients in general perform worse than healthy controls, unmedicated as well as medicated patients (Brody et al., $1980(n=6)$; Braff et al., $1982(n=16)$; Butler et al., 1996 $(n=7)$ and Cadenhead et al., $1997(n=14)$.

There are two hypothetical scenarios for explaining the effects of typical vs. atypical medication. If unmedicated patients performed better than medicated ones including atypically treated patients (like in the contrast detection studies cited above), medication would have deleterious effects on vision, with stronger deterioration for patients with typical medication than atypical medication. In this hypothetical scenario, reduction of positive symptoms would come at the cost of visual deficits. If, on the other extreme, unmedicated patients perform similar to patients with typical medication (like in the backward masking studies mentioned above), atypical medication does not only reduce positive symptoms but also improves vision. Results from contrast detection favor the first scenario, backward masking results the second one. However, samples sizes are, as mentioned, too small to draw proper conclusions and further investigations are needed.

Not only typical vs. atypical medication changes backward masking and contrast sensitivity but also benzodiazepines (e.g., lorazepam) can prolong backward masking dramatically (see Giersch and Herzog, 2004). It might be hypothesized that not the specific type of medication but solely the dose of antipsychotics produces differences in performance. We did not observe differences in CPZ equivalents between both groups and no significant correlation between performance in the studied tests and CPZ. It also might be hypothesized that the differences in performance are due to differences in severity of the psychopathology in both groups. However, psychopathology (BPRS and SANS/SAPS) were not statistically different in both groups.

One important conclusion of our study is that medication needs to be tightly controlled when comparing results from different studies. The effect sizes of typical and atypical medication are substantial (e.g., 0.68 for VD) and, thus, mixed results in the literature may be caused by different ratios of typically vs. atypically medicated patients. One option could be to plot results separately for the two patient groups. However, medication is usually very heterogeneous including many drug combinations and thus collapsing data is not an easy task. Moreover, subdividing antipsychotics into typicals and atypicals is usually an oversimplification since most atypical antipsychotic have different receptor profiles (Li et al., 2016; for example NMDA receptors).

The masking deficits of the schizophrenia patients are well reflected in strongly reduced amplitudes in the EEG of chronic patients (Plomp et al., 2013), first episode patients 
(Favrod et al., 2018) and in students scoring high in cognitive disorganization (Favrod et al., 2017) but not relatives who show even increased amplitudes (da Cruz et al., submitted). Future work needs to show how medication influences EEG. We proposed that the nicotinic receptor $\alpha 7$ subunit gene (CHRNA7) influences backward masking because patients with a specific mutation showed increased masking (Bakanadize et al., 2013; Herzog et al., 2013). In general, it needs to be seen whether medication plays a role only for patients with a specific genetic make up and whether there are gender differences in medication (Shaqiri et al., 2018). Finally, it remains an open question why certain visual tasks, such as crowding (Roinishvili et al., 2015; 2008) and non-retinotopic integration (Lauffs et al., 2016), are not affected by the disease and whether medication plays a different role for these tasks than contrast detection and backward masking. For all these questions, larger sample sizes and meta-analyses are needed.

Our study has the following limitations. First, our findings need to be confirmed by larger studies and with different paradigms. Since the patients were not randomly assigned to groups (typical vs. atypical) we cannot determine whether performance differences are caused primarily by the differences in the drugs or specific patient characteristics (e.g. metabolic, inflammatory problems, and systemic diseases). It is important to note that lessexpensive typical antipsychotic medications can have a higher percentage of prescription in economically less-developed countries (Puyat et al., 2013).

In summary, we found that atypical medication is superior to typical medication for vision. To properly evaluate the effects of medication, studies with unmedicated patients are needed.

\section{Acknowledgements}

The present study followed the ethical principles of the Declaration of Helsinki and was approved by the Committee of Ethics in Research of the Health Sciences Center of Federal University da Paraiba, Brazil, and from the Georgian National Council on Bioethics, Georgia. Written informed consent was obtained from all of the participants. We would like to thank the Coordination for the Improvement of Higher Education Personnel (CAPES).

\section{References}


American Psychiatric Association, 2015. Structured Clinical Interview for DSM-5 (SCID-5). Washington, DC.

American Psychiatric Association, 2013. Diagnostic and Statistical Manual of Mental Disorders (DSM-5®). American Psychiatric Pub. Arlington, VA.

Andreasen, N.C., 1984. Scale for the Assessment of Positive Symptoms (SAPS). University of Iowa, Iowa City

Andreasen, N.C., 1984. Scale for the Assessment of Negative Symptoms (SANS). University of Iowa, Iowa City

Antonius, R., 2003. Interpreting quantitative data with SPSS. SAGE Publications., London, UK.

Bach, M., 1996. The Freiburg Visual Acuity test--automatic measurement of visual acuity. Optom. Vis. Sci. Off. Publ. Am. Acad. Optom. 73, 49-53.

Bakanidze, G., Roinishvili, M., Chkonia, E., Kitzrow, W., Richter, S., Neumann, K., et al., 2013. Association of the nicotinic receptor $\alpha 7$ subunit gene (CHRNA7) with schizophrenia and visual backward masking. Front. Psychiatry. 4, 133. https://doi.org/10.3389/fpsyt.2013.00133

Beasley, T.M., Erickson, S., Allison, D.B., 2009. Rank-based inverse normal transformations are increasingly used, but are they merited? Behav. Genet. 39, 580-595. https://doi.org/10.1007/s10519-009-9281-0

Braff, D.L., Saccuzzo, D.P., 1981. Information processing dysfunction in paranoid schizophrenia: a two-factor deficit. Am. J. Psychiatry 138, 1051-1056. https://doi.org/10.1176/ajp.138.8.1051

Braff, D.L., Saccuzzo, D.P., 1982. Effect of antipsychotic medication on speed of information processing in schizophrenic patients. Am. J. Psychiatry 139, 1127-1130. https://doi.org/10.1176/ajp.139.9.1127

Brisch, R., Saniotis, A., Wolf, R., Bielau, H., Bernstein, H.-G., Steiner, J., et al., 2014. The role of dopamine in schizophrenia from a neurobiological and evolutionary perspective: old fashioned, but still in vogue. Front. Psychiatry 5. https://doi.org/10.3389/fpsyt.2014.00047

Brody, D., Saccuzzo, D.P., Braff, D.L., 1980. Information processing for masked and unmasked stimuli in schizophrenia and old age. J. Abnorm. Psychol. 89, 617-622.

Buchsbaum, M.S., Nuechterlein, K.H., Haier, R.J., Wu, J., Sicotte, N., Hazlett, E., et al., 1990. Glucose metabolic rate in normals and schizophrenics during the continuous performance test assessed by positron emission tomography. Br. J. Psychiatry 156 (2), 216-227. doi:10.1192/bjp.156.2.216

Butler, P.D., Harkavy-Friedman, J.M., Amador, X.F., Gorman, J.M., 1996. Backward masking in schizophrenia: relationship to medication status, neuropsychological functioning, and dopamine metabolism. Biol. Psychiatry 40, 295-298. https://doi.org/10.1016/0006-3223(96)00007-8

Cadenhead, K.S., Dobkins, K., McGovern, J., Shafer, K., 2013. Schizophrenia spectrum participants have reduced visual contrast sensitivity to chromatic (red/green) and luminance (light/dark) stimuli: new insights into information processing, visual channel function, and antipsychotic effects. Front. Psychol. 4, 535. https://doi.org/10.3389/fpsyg.2013.00535

Cadenhead, K.S., Geyer, M.A., Butler, R.W., Perry, W., Sprock, J., Braff, D.L., 1997. Information processing deficits of schizophrenia patients: relationship to clinical ratings, gender and medication status. Schizophr. Res. 28, 51-62. https://doi.org/10.1016/S0920-9964(97)00085-6

Chen, Y., Levy, D.L., Sheremata, S., Nakayama, K., Matthysse, S., Holzman, P.S., 2003. Effects of typical, atypical, and no antipsychotic drugs on visual contrast detection in 
schizophrenia. Am. J. Psychiatry 160, 1795-1801. https://doi.org/10.1176/appi.ajp.160.10.1795

Chkonia, E., Roinishvili, M., Makhatadze, N., Tsverava, L., Stroux, A., Neumann, K., et al., 2010. The shine-through masking paradigm is a potential endophenotype of schizophrenia. PloS One 5, e14268. https://doi.org/10.1371/journal.pone.0014268

da Cruz, J.R., Shaqiri, A., Roinishvili, M., Chkonia, E., Brand, A., Figueiredo, P., Herzog, M.H., submitted. Neural compensation mechanisms of siblings of schizophrenia patients as revealed by high-density EEG.

Désaméricq, G., Schurhoff, F., Meary, A., Szöke, A., Macquin-Mavier, I., Bachoud-Lévi, A.C., et al., 2014. Long-term neurocognitive effects of antipsychotics in schizophrenia: a network meta-analysis. Eur J Clin Pharmacol. 70(2):127-34. doi: 10.1007/s00228-013-1600-y.

Eriksen, B.A., Eriksen, C.W., 1974. Effects of noise letters upon the identification of a target letter in a nonsearch task. Percept. Psychophys. 16, 143-149. https://doi.org/10.3758/BF03203267

Favrod, O., Sierro, G., Roinishvili, M., Chkonia, E., Mohr, C., Herzog, M.H. et al., 2017. Electrophysiological correlates of visual backward masking in high schizotypic personality traits participants. Psychiatry Res, 254, 251-257. https://doi.org/10.1016/j.psychres.2017.04.051

Favrod, O., Roinishvili, M., da Cruz, J.R., Brand, A., Okruashvili, M., Gamkrelidze, T., et al., 2018. Electrophysiological correlates of visual backward masking in patients with first episode psychosis. Psychiatry Research: Neuroimaging, 282, 64-72. https://doi.org/10.1016/j.pscychresns.2018.10.008

Fernandes, T.M.P., Almeida, N.L. de, Santos, N.A. dos, 2017a. Effects of smoking and smoking abstinence on spatial vision in chronic heavy smokers. Sci. Rep. 7, 1690. https://doi.org/10.1038/s41598-017-01877-z

Fernandes, T.M.P., Andrade, S.M., Andrade, M.J.O., Nogueira, R.M.T.B.L., Santos, N.A., 2017b. Colour discrimination thresholds in type 1 bipolar disorder: a pilot study.

Scientific Reports 7, 16405. https://doi.org/10.1038/s41598-017-16752-0

Fernandes, T.M.P., Souza, R.M. da C. e, Santos, N.A. dos, 2018a. Visual function alterations in epilepsy secondary to migraine with aura: a case report. Psychol. Neurosci. 11, 8694. https://doi.org/10.1037/pne0000121

Fernandes, T.M.P., Andrade, M.J.O. de, Santana, J.B., Nogueira, R.M.T.B.L., Santos, N.A. dos, 2018b. Tobacco use decreases visual sensitivity in schizophrenia. Front. Psychol. 9. https://doi.org/10.3389/fpsyg.2018.00288

Fernandes, T.P., Silverstein, S.M., Almeida, N.L., Santos, N.A., 2018c. Visual impairments in tobacco use disorder. Psychiatry Res. 271, 60-67. https://doi.org/10.1016/j.psychres.2018.11.024

Fernandes, T.M.P., Silverstein, S.M., Butler, P.D., Kéri, S., Santos, L.G., Nogueira, R.L., et al., 2018d. Color vision impairments in schizophrenia and the role of antipsychotic medication type. Schizophr. Res. https://doi.org/10.1016/j.schres.2018.09.002

Fioravanti, M., Bianchi, V., Cinti, M.E., 2012. Cognitive deficits in schizophrenia: an updated metanalysis of the scientific evidence. BMC psychiatry, 12, 64. doi:10.1186/1471-244X-12-64.

Giersch, A., Herzog, M.H., 2004. Lorazepam strongly prolongs visual information processing. Neuropsychopharmacology, 29(7), doi:10.1038/sj.npp.1300429

1386-1394.

Green, M.F., Nuechterlein, K.H., Mintz, J., 1994. Backward masking in schizophrenia and mania. I. Specifying a mechanism. Arch. Gen. Psychiatry 51, 939-944. 
Jablensky, A., 2010. The diagnostic concept of schizophrenia: its history, evolution, and future prospects. Dialogues Clin. Neurosci. 12, 271-287.

Hagger, C., Buckley, P., Kenny, J.T., Friedman, L., Ubogy, D., Meltzer, H.Y., 1993. Improvement in cognitive functions and psychiatric symptoms in treatment-refractory schizophrenic patients receiving clozapine. Biol. Psychiatry. 34:702-712.

Harvey, P.D., Keefe, R.S.E., Moskowitz, J., Putnam, K.M., Mohs, R.C., Davis, K.L., 1990. Attentional markers of vulnerability to schizophrenia: performance of medicated and unmedicated patients and normals. Psychiatry Research, 33(2), 179-188. doi:10.1016/0165-1781(90)90072-d

Harvey, P.D., Keefe, R.S., 2001. Studies of cognitive change in patients with schizophrenia following novel antipsychotic treatment. Am J Psychiatry. 158(2):176-84.

Heatherton, T.F., Kozlowski, L.T., Frecker, R.C., Fagerstorm, K.O., 1991. The Fagerström test for nicotine dependence: a revision of the Fagerstrom tolerance questionnaire. British journal of addiction, 86(9), 1119-1127.

Herzog, M.H., Kopmann, S., Brand, A., 2004. Intact figure-ground segmentation in schizophrenia. Psychiatry Res., 129(1), 55-63. https://doi.org/10.1016/j.psychres.2004.06.008

Herzog, M.H., Roinishvili, M., Chkonia, E. Brand, A., 2013. Schizophrenia and visual backward masking: a general deficit of target enhancement. Frontiers in Psychol., 4, 254. https://doi.org/10.3389/fpsyg.2013.00254

Herzog, M.H., Brand, A., 2015. Visual masking and schizophrenia. Schizophrenia Research: Cognition, 2(2), 64-71. https://doi.org/10.1016/j.scog.2015.04.001

Keefe, R.S., Sweeney, J.A., Gu, H., Hamer, R.M., Perkins, D.O., McEvoy, J.P., et al., 2007a. Effects of olanzapine, quetiapine, and risperidone on neurocognitive function in early psychosis: a randomized, double-blind 52-week comparison. Am J Psychiatry. 164(7):1061-71.

Keefe, R.S., Bilder, R.M., Davis, S.M., Harvey, P.D., Palmer, B.W., Gold, J.M., et al., 2007b. Neurocognitive effects of antipsychotic medications in patients with chronic schizophrenia in the CATIE Trial. Arch Gen Psychiatry. 64(6):633-47.

Kéri, S., Antal, A., Szekeres, G., Benedek, G., Janka, Z., 2002. Spatiotemporal visual processing in schizophrenia. J. Neuropsychiatry Clin. Neurosci. 14, 190-196. https://doi.org/10.1176/jnp.14.2.190

Kiss, I., Fábián, A., Benedek, G., Kéri, S., 2010. When doors of perception open: visual contrast sensitivity in never-medicated, first-episode schizophrenia. J. Abnorm. Psychol. 119, 586-593. https://doi.org/10.1037/a0019610

Lauffs, M.M., Shaqiri, A., Brand, A., Roinishvili, M., Chkonia, E., Öğmen, H. et al., 2016. Local versus global and retinotopic versus non-retinotopic motion processing in schizophrenia patients. Psychiatry Res., 246, 461-465. https://doi.org/10.1016/j.psychres.2016.09.049

Lee, M.A., Jayathilake, K., Meltzer, H.Y., 1997. A comparison of the effect of clozapine with typical neuroleptics in cognitive function in neuroleptic-responsive schizophrenia. Schizophr Res. 1-11.

Levitt, H., 1971. Transformed up-down methods in psychoacoustics. J. Acoust. Soc. Am. 49, Suppl 2:467+

Li, P., Snyder, G.L., Vanover, K.E., 2016. Dopamine targeting drugs for the treatment of schizophrenia: past, present and future. Current topics in medicinal chemistry, 16(29), 3385-3403. 
Liu, S., 2000. Effects of atypical neuroleptics on sustained attention deficits in schizophrenia a trial of risperidone versus haloperidol. Neuropsychopharmacology, 22(3), 311-319. doi:10.1016/s0893-133x(99)00137-2

Mathers, C.D., Loncar, D., 2006. Projections of global mortality and burden of disease from 2002 to 2030. PLOS Med 3, https://doi.org/10.1371/journal.pmed.0030442

Meltzer, H.Y., 2017. New trends in the treatment of schizophrenia. CNS Neurol. Disord. Drug Targets 16, 900-906. https://doi.org/10.2174/1871527316666170728165355

Nestor, P.G., Faux, S.F., McCarley, R.W., Sands, S.F., Horvath, T.B., Peterson, A., 1991. Neuroleptics improve sustained attention in schizophrenia. A study using signal detection theory. Neuropsychopharmacology. 4(2):145-9.

Orzack, M.H., Kornetsky, C., Freeman, H., 1967. The effects of daily administration of carphenazine on attention in the schizophrenic patient. Psychopharmacologia. 11(1):31-38

Plomp, G., Roinishvili, M., Chkonia, E., Kapanadze, G., Kereselidze, M., Brand, A. et al., 2012. Electrophysiological evidence for ventral stream deficits in schizophrenia patients. Schizophrenia https://doi.org/10.1093/schbul/sbr175

bulletin, 39(3),

547-554.

Puyat, J. H., 2013. Is the influence of social support on mental health the same for immigrants and non-immigrants? Journal of Immigrant and Minority Health, 15(3), 598-605.

Roinishvili, M., Cappe, C., Shaqiri, A., Brand, A., Rürup, L., Chkonia, E., Herzog, M.H., 2015. Crowding, grouping, and gain control in schizophrenia. Psychiatry Res. 226, 441-445. https://doi.org/10.1016/j.psychres.2015.01.009

Roinishvili, M., Chkonia, E., Brand, A., Herzog, M.H., 2008. Contextual suppression and protection in schizophrenic patients. European archives of psychiatry and clinical neuroscience, 258(4), 210-216. DOI 10.1007/s00406-007-0780-9

Shaqiri, A., Willemin, J., Sierro, G., Roinishvili, M., Iannantuoni, L., Rürup, L., et al., 2015. Does chronic nicotine consumption influence visual backward masking in schizophrenia and schizotypy? Schizophr. Res. Cogn. 2, 93-99. https://doi.org/10.1016/j.scog.2015.04.006

Shaqiri, A., Roinishvili, M., Grzeczkowski, L., Chkonia, E., Pilz, K., Mohr, C., et al., 2018. Sex-related differences in vision are heterogeneous. Scientific reports, 8(1), 7521. DOI: $10.1038 / \mathrm{s} 41598-018-25298-8$

Shoshina, I. I., Shelepin, Y. E., Semenova, N. B., 2014. Frequency-contrast sensitivity of visual stimulus perception in patients with schizophrenia treated with atypical and typical antipsychotics. Human Physiology, 40(1), 35-39.

Shoshina, I. I., Shelepin, Y. E., 2015. Contrast sensitivity in patients with schizophrenia of different durations of illness. Neuroscience and Behavioral Physiology, 45(5), 512516.

Silverstein, S.M., 2016. Visual perception disturbances in schizophrenia: a unified model. Neb. Symp. Motiv. Neb. Symp. Motiv. 63, 77-132.

Slaghuis, W.A., 1998. Contrast sensitivity for stationary and drifting spatial frequency gratings in positive- and negative-symptom schizophrenia. J. Abnorm. Psychol. 107, 49-62. https://doi.org/10.1037/0021-843X.107.1.49

Stroop, J.R., 1935. Studies of interference in serial verbal reactions. J. Exp. Psychol. 18, 643662. https://doi.org/10.1037/h0054651

Tabachnick, B.G., Fidell, L.S., 2007. Using multivariate statistics, 5th ed. Allyn and Bacon/Pearson Education, Boston, MA. 
Tombaugh, T.N., 2004. Trail Making Test A and B: normative data stratified by age and education. Arch. Clin. Neuropsychol. 19, 203-214. https://doi.org/10.1016/S08876177(03)00039-8

Woodward, N.D., Purdon, S.E., Meltzer, H.Y., Zald, D.H., 2005. A meta-analysis of neuropsychological change to clozapine, olanzapine, quetiapine, and risperidone in schizophrenia. Int J Neuropsychopharmacol. 8(3):457-72. 
Figure Captions

Figure 1. Contrast sensitivity function task. The task was to identify, using a remote control response box, whether the gratings were presented either on the left or right side of the computer screen. Each stimulus had an exposure time of $600 \mathrm{~ms}$, with an inter-trial interval of $300 \mathrm{~ms}$. The Metropsis algorithm randomizes spatial frequencies (low, medium and high) and contrast values.

Figure 2. Contrast sensitivity as a function of spatial frequency (cpd) in healthy controls and schizophrenia patients taking atypical and typical antipsychotics, respectively. The data are shown as the mean sensitivity (reciprocal of contrast threshold). Error bars represent the standard errors of the mean.

Figure 3. Differences between the three groups for three cognitive tests. (A) Mean reaction time (s) for the Mixed-Flanker task; (B) Mean reaction time (s) for the Stroop task comparing differences for the congruent vs. incongruent responses; (C) Mean reaction time for the Trail-Making Test-B completion time. The data are presented as means and standard error (SEM) of the means.

Figure 4. (A) We first presented a Vernier alone (A) and as a second step (B), this Vernier was followed by a blank screen (ISI) and a grating comprised of 25 (or 5, not presented) aligned Verniers (SOA=VD + ISI).

Figure 5. Performance on Vernier Duration (VD), Stimulus-Onset-Asynchrony 25 (SOA25) and Stimulus-Onset-Asynchrony 5 (SOA5). Results are displayed as means and standard errors of the mean (error bars). All three tests revealed significant differences between SCZ

Figure 6. Performance for the cognitive tests (Continuous Performance Test (CPT) and Wisconsin Card Sorting Test (WCST) is higher in patients treated with atypical than with typical drugs. These differences, however, are not significant. Both groups of patients performed significantly worse than healthy controls. Results are indicated as means and standard errors. 